\title{
Paper
}

\section{Development of Lightweight Compressed 8K UHDTV over IP Transmission Device Realizing Live Remote Production}

\author{
Junichiro Kawamoto ${ }^{\dagger}$ (member), Tomofumi Koyama ${ }^{\dagger}$ (member), Masahiro Kawaragi ${ }^{\dagger}$ (member), \\ Kyoichi Saito ${ }^{\dagger}$ (member) and Takuya Kurakake ${ }^{\dagger}$ (member)
}

\begin{abstract}
Studies on live program production systems using Internet Protocol (IP) communications technology at broadcast stations are progressing. Remote production is attracting attention as a new style of live program production using IP. In remote production, broadcast stations and venues are connected by IP network, and programs are remotely produced from the broadcast station side. To enable remote production, it is necessary for both the venue and the broadcast station to share, in real-time, high-quality video taken at the venue. It is also required to bidirectionally communicate signals other than video/audio that are necessary for program production, such as control and communication line signals. To realize $8 \mathrm{~K}$ remote production, we have developed a lightweight compressed $8 \mathrm{~K}$ over IP transmission device. In this work, we describe its functions and report experimental results on multi-channel audio remote production with $8 \mathrm{~K}$ video and real-time $8 \mathrm{~K}$ camera control on a $1000-\mathrm{km}$ IP network.
\end{abstract}

Keywords: 8K UHDTV over IP, lightweight compression, remote production.

\section{Introduction}

In recent years, the spread of flexible and efficient networks using Internet Protocol (IP) communication technology has been accelerating worldwide due to an increase in Ethernet ${ }^{1)}$ speed and decrease in the cost per bit $^{2)}$. The field of program production in broadcast stations is no exception, and studies on program production systems using IP technology in broadcast stations are being actively conducted. In particular, in live program production systems, standardization related to uncompressed video transmission over IP signals has been achieved ${ }^{3), 4)}$. As a result, the implementation of live program production systems at broadcast stations using transmission technology called Video over IP or Media over IP has shown great progress $^{5)-8)}$.

In terms of video signals, $4 \mathrm{~K}$ and $8 \mathrm{~K}$ ultra-high definition television (UHDTV), which evolved from the earlier 2K HDTV broadcasting, are being developed for next-generation television services ${ }^{9}$. These video resolutions are 3,840 $(\mathrm{H}$ : horizontal $) \times 2,160(\mathrm{~V}$ : vertical $)$

Received July 1, 2019; Revised November 28, 2019; Accepted December 4, 2019

†Advanced Transmission Systems Research Division, Science \& Technology Research Laboratories, NHK.

(Tokyo, Japan) for $4 \mathrm{~K}$ and $7,680(\mathrm{H}) \times 4,320(\mathrm{~V})$ for $8 \mathrm{~K}$. In Japan, test broadcasting using satellites has already begun from 2016, and from December 2018, the world's first $8 \mathrm{~K}$ broadcasting service has been launched10). $8 \mathrm{~K}$ provides ultra-realistic viewing experiences to viewers, creating the sensation that they are in the actual scene. Because an uncompressed $8 \mathrm{~K}$ video has an extremely high data rate-specifically, $40 \mathrm{~Gb} / \mathrm{s}$ (in the case of $60-\mathrm{Hz}$ progressive video frame rate, 4:2:2 sampling structure, and 10-bit pixel depth) and $144 \mathrm{~Gb} / \mathrm{s}$ (in the case of 120$\mathrm{Hz}$ progressive video frame rate, 4:4:4 sampling structure, and 12-bit pixel depth)-it is important to efficiently migrate from $2 \mathrm{~K}$ broadcasting services to $8 \mathrm{~K}$ broadcasting services. As a method to do so, an $8 \mathrm{~K}$ program production system using IP communication technology can be considered.

Serial digital interface (SDI) ${ }^{11)}$ signals are frequently used in the program production systems of current broadcast stations. SDI has been used for many years as a signal interface standard that can continuously deliver high quality and stable broadcasting service. As a representative SDI signal, it consists of high definitionSDI (HD-SDI) ${ }^{12)}$ signals with a transmission speed of 1.5 $\mathrm{Gb} / \mathrm{s}$ used in the $2 \mathrm{~K}$ broadcasting service, and in recent years, faster $12 \mathrm{G}-\mathrm{SDI}^{13}$ ) signals have also been standardized. An HD-SDI can transmit one 2K HDTV 
video channel and a $12 \mathrm{G}-\mathrm{SDI}$ can transmit one $4 \mathrm{~K}$ UHDTV video channel. These SDI signals follow a oneto-one and unidirectional transmission standard where one kind of signal is transmitted on one coaxial cable. Therefore, when SDI signals are used for $4 \mathrm{~K} / 8 \mathrm{~K}$ broadcasting services, the program production system becomes complicated when connecting a large number of coaxial cables because of the extremely high data rate. Moreover, if additional signal transmissions are needed, more cables need to be laid, so the system lacks scalability.

IP communication technology has features such as bidirectionality and ease of multiplexing. In recent years, a 400-Gb/s Ethernet transmission standard ${ }^{14)}$ has been completed, and the speeding up of Ethernet devices along with the lowering costs due to commoditization is nothing short of remarkable. Therefore, IP communication technology has a very high affinity for efficiently realizing a wide variety of broadcasting services.

Furthermore, introducing IP technology to broadcast stations not only makes the transmission more efficient but also has the potential to provide a flexible new work style in terms of the operation of the program production system. One example of this is a remote production that produces live programs from the broadcast station side by connecting venues and the broadcast station via IP network. In the conventional program production system, many broadcast engineers were producing programs on the venue side. In the remote production system, live videos at the venue side are shared between the venue and the broadcast station in real time, and engineers can operate from the broadcast station side, so they do not have to physically go to the venue. The system can also change between venues instantaneously. This is how efficient program production can be realized by the remote production system. However, there is one technical problem: it is necessary to transmit video with high image quality and low delay, and since $8 \mathrm{~K}$ has an extremely high data rate, an efficient transmission method is needed. There are some related works on remote production ${ }^{15)-17)}$, but they are basically limited to $2 \mathrm{~K}$ video transmissions and do not consider $8 \mathrm{~K}$ remote production. Since the transmission data rate of $8 \mathrm{~K}$ has become extremely high, in order to realize remote production of $8 \mathrm{~K}$, we need to find a way to efficiently transmit high image quality video with low delay from the venue to the broadcast station.

Therefore, we have developed a lightweight compressed 8K UHDTV over IP transmission device that achieves efficient and flexible program production using IP communication technology. Up to now, uncompressed $2 \mathrm{~K}$ and $4 \mathrm{~K}$, and their compressed over IP transmission devices development has been conducted ${ }^{18), 19)}$. Although 8K UHDTV lightweight compression technology exists, there is no device that transmits $8 \mathrm{~K}$ with high image quality and low latency with various material signals in both directions using a general purpose $10 \mathrm{~Gb} / \mathrm{s}$ Ethernet IP line. There are $8 \mathrm{~K}$ UHDTV over IP transmission devices for a remote production usage, but these transmission bandwidth requires $100 \mathrm{~Gb} / \mathrm{s}$ or 40 Gb/s Ethernet IP line, so efficient transmission cannot be achieved. By using the proposed device, long-distance $8 \mathrm{~K}$ remote production can be realized with efficiently 10 Gb/s Ethernet IP line. In this paper, we explain the functions of the proposed device and discuss the results of a remote production experiment to determine whether it can share $8 \mathrm{~K}$ video with high image quality and low delay in real time even in a remote place.

\section{8K Remote Production}

\subsection{Remote Production}

A conceptual diagram comparison between conventional production and remote production is shown in Fig. 1. Conventionally, in live program production, a lot of production equipment is installed in large-scale outside-broadcasting (OB) vans, and broadcasting engineers perform almost all of the video switching and audio mixing in these vans on the venue side. Switched and mixed signals are transmitted to the broadcast station as SDI signals, which are generally broadcast after keying (such as adding captions) from the broadcast station. In contrast, in remote production, there is just one device that transmits video/audio, and minimal engineers on the venue side, and switching and mixing is mainly done on the broadcast station side. If all that is required is a transmission line, it is possible to produce the bulk of a program on the broadcast station side. Thus, efficient and flexible broadcasting program productione.g., reduced travel expenses, improved utilization of program production equipment, the implementation of live program production from a narrow place that largescale $O B$ vans cannot enter, and smooth switching between multiple venues-should be possible.

\subsection{Requirements for Proposed Device}

In order to enable remote production, there are two conditions that should be fulfilled. First, high-quality video taken at the venue should be shared between the venue and the broadcast station in real time. Second, 


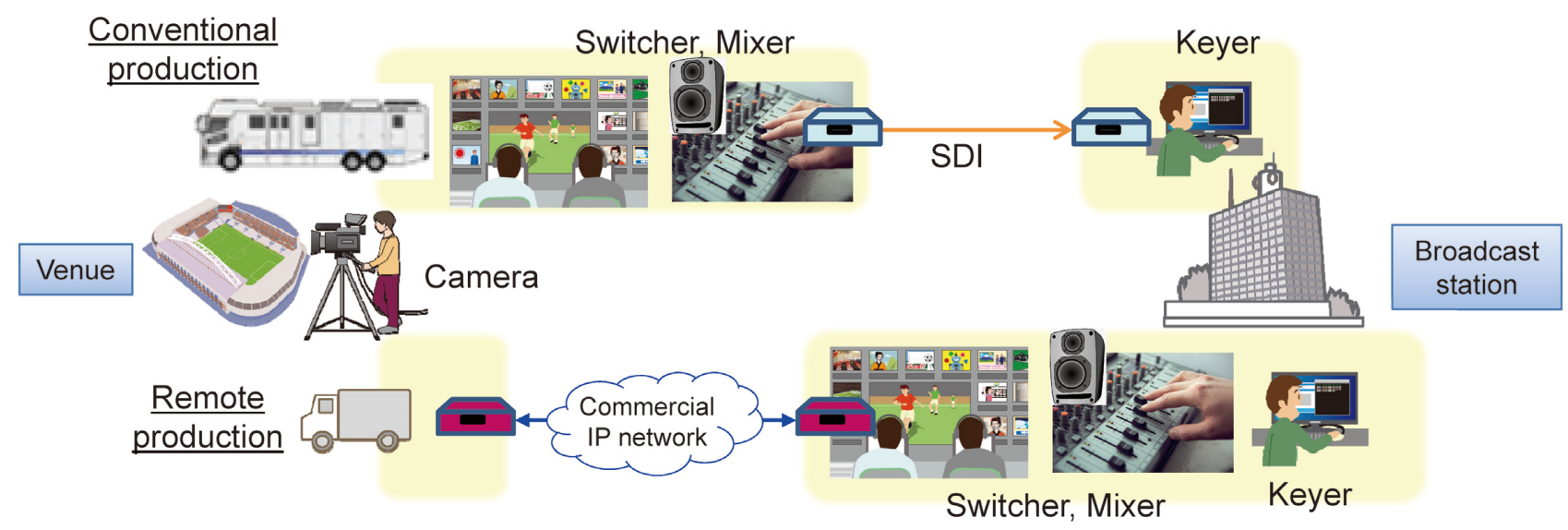

Fig.1 Conceptual diagram comparison between "conventional production" and "remote production"

the signals necessary for program production other than video/audio, such as control and communication lines, should be bidirectionally communicated. To realize $8 \mathrm{~K}$ remote production, we set the following requirements for our $8 \mathrm{~K}$ transmission device.

1) $8 \mathrm{~K}$ program video is efficiently transmitted with high image quality and low latency.

2) IP format signal is transmitted using $10-\mathrm{Gb} / \mathrm{s}$ Ethernet transmission (10-GbE) line.

Regarding the first requirement, to efficiently transmit high data rate $8 \mathrm{~K}$ program video in as narrow band as possible, we decided to use lightweight video compression technology. For video quality, peak signalto-noise ratio (PSNR) set to $40 \mathrm{~dB}$ was used as a guide. This technology can achieve visually lossless video compared with the original video with intra-frame compression. As for latency, we aimed to achieve an $8 \mathrm{~K}$ video processing time on the order of milliseconds. While there are several video compression techniques that use inter-frame compression to increase compression efficiency, lightweight video compression technology with intra-frame compression is effective in achieving the aforementioned visually lossless and low latency. Remote production aims to eliminate the location constraints of the program production environment by sharing videos in real time with the venue and the broadcast station. As an example of remote production ${ }^{17)}$, it sets the delay requirement in remote production in mobile environment to $50 \mathrm{~ms}$. In the case of $60-\mathrm{Hz}$ progressive video frame rate, its value corresponds to approximately three video frames. It is difficult to realize real-time video sharing between the venue and the broadcast station, especially in scenes where video changes greatly in several frames, such as sports event program production. Therefore, the target total-delay in a single direction of real-time remote production was set to one video frame time (e.g., $16.7 \mathrm{~ms}$
@60-Hz frame) or less, without packet jitter absorption buffer.

Regarding the second requirement, to communicate various signals bidirectionally, the transmission signal was multiplexed into IP signals and targeted to be able to transmit using one or two 10-GbE lines. As a result of investigating the services of various telecommunications carriers in Japan, the 10-GbE line is known to carry out a wide range of services nationwide by most telecommunications carriers, and in the future, it is expected to excel in terms of line availability and to decline in cost due to competition. The 10-GbE line covered in this paper is a line guaranteed quality, but if it can realize the same higher quality, it can be used on the public Internet.

Up to now, although $8 \mathrm{~K}$ UHDTV lightweight compression technology exists, there is no device that transmits $8 \mathrm{~K}$ with high image quality and low latency with IP format signal using $10-\mathrm{GbE}$ transmission line. Therefore, we propose a lightweight compressed 8K over 10-GbE transmission line.

\section{Proposed Lightweight Compressed 8K over IP Transmission Device}

\subsection{Configuration}

Figure 2 shows a configuration of the lightweight compressed 8K over IP transmission device. Figure 2(a) shows the $8 \mathrm{~K} 60-\mathrm{Hz}$ video frame $(8 \mathrm{~K} 60 \mathrm{p})$ transmission device, and Fig. 2(b) shows the $8 \mathrm{~K} 120-\mathrm{Hz}$ video frame (8K120p) transmission device. As a basic configuration, the SDI signal is input to the transmitter and output from the receiver, and the constant bit rate IP format signal is transmitted over the Ethernet transmission line. By applying lightweight compression technology to video signals, high-image-quality and low-latency transmission is realized while reducing transmission bandwidth. The signal specifications are listed in Table 


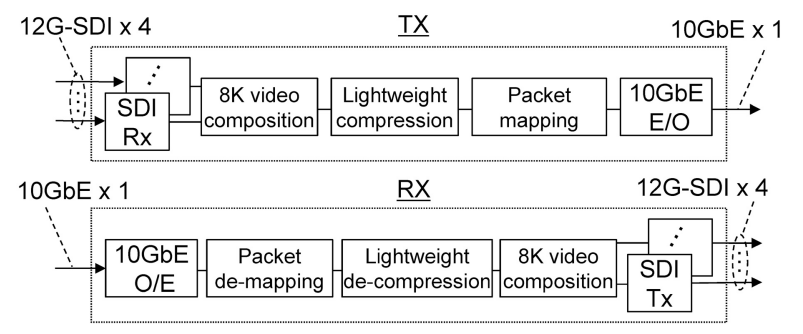

(a) $8 \mathrm{~K} 60 \mathrm{p}$

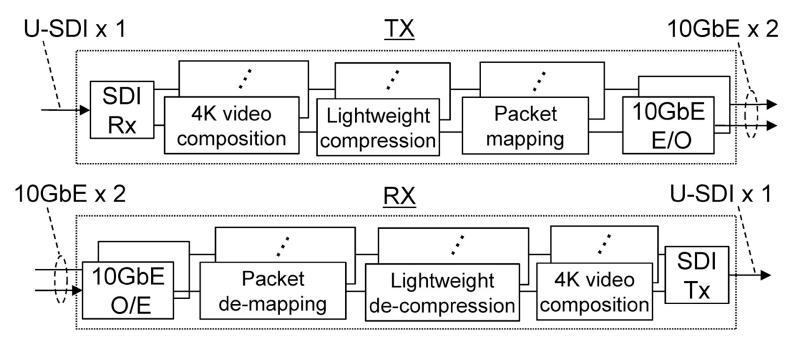

(b) $8 \mathrm{~K} 120 \mathrm{p}$

Fig.2 Configuration of proposed device

Table 1 Signal Specifications

\begin{tabular}{l|c|c}
\hline \hline Video format & $\begin{array}{c}8 \mathrm{~K} 60 \mathrm{p} \\
(4: 2: 2,10 \mathrm{bit})\end{array}$ & $\begin{array}{c}8 \mathrm{~K} 120 \mathrm{p} \\
(4: 4: 4,12 \mathrm{bit})\end{array}$ \\
\hline SDI interface & $12 \mathrm{G}-\mathrm{SDI} \times 4$ & $\mathrm{U}-\mathrm{SDI} \times 1$ \\
\hline Compression technology & \multicolumn{2}{|c}{$\mathrm{TICO}$} \\
\hline Compression ratio & \multicolumn{2}{|c}{$1 / 5 \cdot 1 / 9$} \\
\hline $\begin{array}{c}\text { Transmission bandwidth } \\
\text { (Ex.) }\end{array}$ & $\begin{array}{c}40 \mathrm{~Gb} / \mathrm{s} \rightarrow 8 \mathrm{~Gb} / \mathrm{s} \\
(\text { Compression ratio: } 1 / 5)\end{array}$ & $\begin{array}{c}144 \mathrm{~Gb} / \mathrm{s} \rightarrow 16 \mathrm{~Gb} / \mathrm{s} \\
(\text { Compression ratio: } 1 / 9)\end{array}$ \\
\hline Packet format & \multicolumn{2}{|c}{ PTP, Black $\cdot$ Burst } \\
\hline Synchronization & $10 \mathrm{GbE} \times 1$ & $10 \mathrm{GbE} \times 2$ \\
\hline Ethernet interface & \multicolumn{2}{|c|}{}
\end{tabular}
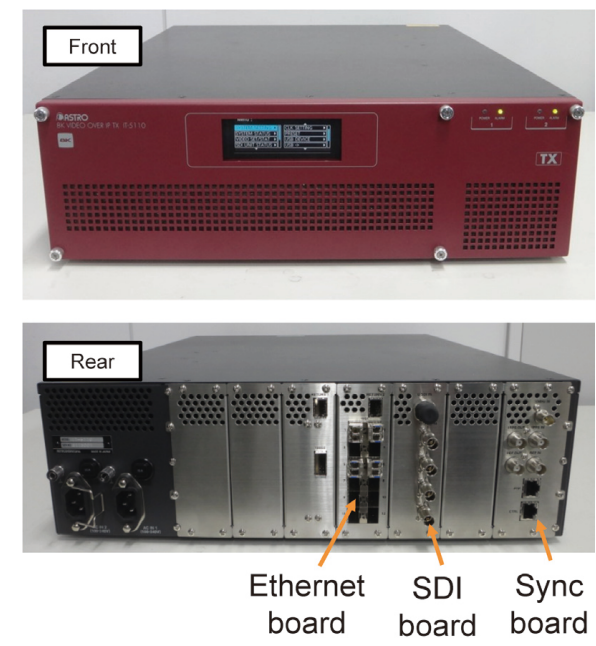

Fig.3 Appearance of developed device

1. Figure 3 shows the appearance of the developed device, which is $3 \mathrm{U}$ in height. In addition to the $12 \mathrm{G}-\mathrm{SDI}$ and U-SDI interfaces, it has multiple ports of $10 \mathrm{GbE}$, and it is possible to transmit not only lightweight compressed $8 \mathrm{~K}$ video but also audio and communication signals by IP format. The power supply is duplexed assuming actual operation. Note that this device is a research prototype, and we plan to further reduce the size after reviewing the substrate configuration.

\subsection{Video Signal Input and Output Interface}

The video signal input and output interface differs between $8 \mathrm{~K} 60 \mathrm{p}$ and $8 \mathrm{~K} 120 \mathrm{p}$. 8K60p is composed of four 12G-SDI signals (Quad-Link 12G) based on SMPTE ST $2082-12^{20)}$. $8 \mathrm{~K} 120 \mathrm{p}$ is composed of an ultrahighdefinition signal/data interface (U-SDI) cable using multi optical fiber based on ITU-R BT.2077 Part $3^{21)}$.

\subsection{Lightweight Compression and}

\section{De-compression}

The lightweight compression technology is characterized by high image quality and low latency, small circuit scale, no need for an external memory, and low power consumption. Although there are several light compression methods, basically each method adopts almost the same algorithm using discrete wavelet transform (DWT). There are three major lightweight compression methods: low latency video codec (LLVC) ${ }^{22)}, \mathrm{VC}-2^{23)}$, and tiny codec $(\mathrm{TICO})^{24)}$. LLVC has been proposed by Sony Corporation, $\mathrm{VC}-2$ by $\mathrm{BBC}$, and $\mathrm{TICO}$ by IntoPIX.SA. TICO standardization is progressing under the name JPEG-XS ${ }^{25}$ ) in the joint photographic experts group (JPEG). With this technology, since compression and de-compression is performed by DWT in units of several dozen lines in an intra frame, the compression ratio itself is not large. However, processing with high image quality and low latency of 1 millisecond or less is possible, and the circuit scale is very small. These features are effective for miniaturization of the device.

A method to map the TICO compressed signal to RTP payload is disclosed ${ }^{24)}$. Also, a method to map the JPEGXS compressed signal to RTP payload is currently being discussed in the internet engineering task force (IETF). New packet mapping method described in the next section is applied to the RFC draft currently issued ${ }^{26)}$.

In $8 \mathrm{~K} 60 \mathrm{p}, 8 \mathrm{~K}$ video composed of four $12 \mathrm{G}-\mathrm{SDI}$ signals is lightweight compressed. The compression ratio can be set from $1 / 5$ to $1 / 9$. Part of these compression ratios are values set by checking that the PSNR value is about 40 dB by video-quality evaluation beforehand. For evaluation images, video-images of horse races, water balls, and marathons from the ultra-high definition/wide-color-gamut standard test sequences series $\mathrm{B}^{27)}$ were used, along with original content. In $8 \mathrm{~K} 120 \mathrm{p}$, four parallel processes are performed using a $4 \mathrm{~K} 120 \mathrm{p}$ TICO circuit. Parallel processing is used because the TICO circuit for $8 \mathrm{~K} 120 \mathrm{p}$ could not be obtained. Since the main compression technique is capable of high image quality processing, even if $8 \mathrm{~K}$ video is compressed and decoded by $4 \mathrm{~K} 4$-parallel processing, it has little effect on the restored video. 


\subsection{Proposed Packet Mapping}

In this device, a proposed packet mapping method that reduces image degradation due to signal quality degradation is applied ${ }^{28)}$. Figure 4(a) compares the conventional and proposed RTP payload mapping methods. Lightweight compression technology divides one video frame into several compression units and compresses each by DWT line-by-line. In Fig. 4(a), lightweight compression is performed on each of the three compression units. The signal after the lightweight compression is called a slice, and the slice signal is mapped to the packet. Each slice signal has different data size. In the conventional mapping, slice signals are mapped sequentially to fixed-length packets without considering the slice boundary. The last slice signal of the video frame is mapped to a packet of an appropriate length. That is, mapping of different slice signals to the same packet is permitted, and the packet length varies within the video frame. In contrast, in the proposed mapping, different slice signals are mapped to different packets, and padding is added so that all packets have the same packet length. The reason for adopting the fixed-length packets is that it is easy to implement the exclusive OR-based FEC ${ }^{29)}$. The exclusive OR-based FEC is a simple error correction technology for high-speed data rates with the wired transmission system. Based on this consideration, this device applied the exclusive OR-based FEC. As shown in Fig. 4(b), when a packet on the slice boundary is lost (e.g., packet no. 2), in the conventional mapping, image degradation is observed over the two compression units with which the lost packet is related (e.g., compression units 0 and 1). When error concealment is performed, the image deteriorates over a wide range, and the PSNR deteriorates. In contrast, in the proposed mapping, the range of image degradation is limited to only the compression unit (e.g., compression unit 0) included in the lost packet. When error concealment is performed, the image is limited to degradation in a relatively narrow range, and the PSNR is increased. The reason such deterioration appears is that, since the slice signal is generated by performing line-by-line wavelet transformation in units of compression unit, past errors in the slice signal are propagated. In the proposed mapping, although data rate is expected to increase due to the addition of padding, the effect on the overall transmission rate is considered negligible. As an example, using an actual compressed signal with 1/5 compression ratio of the Ship's 8K image shown in Fig. 4(c), the proposed mapping generates 20773 packets per frame,

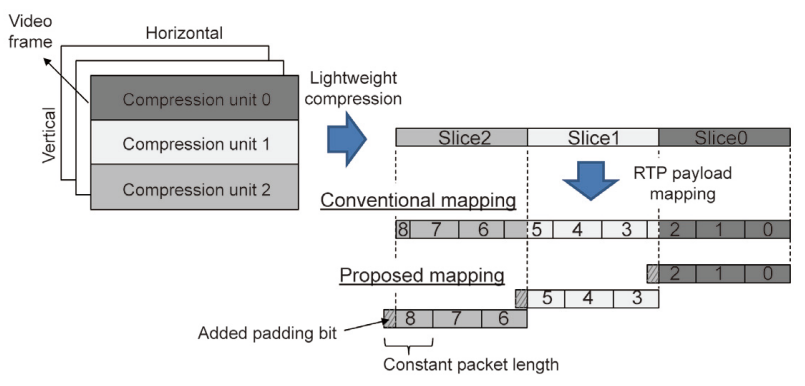

(a) Mapping method

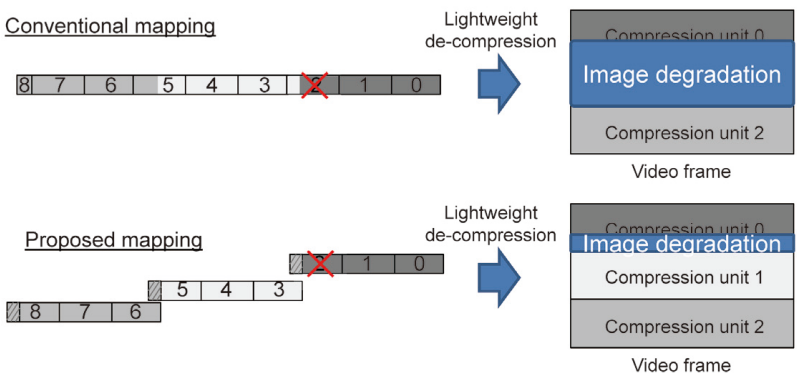

(b) Effect of packet loss on lightweight de-compressed video
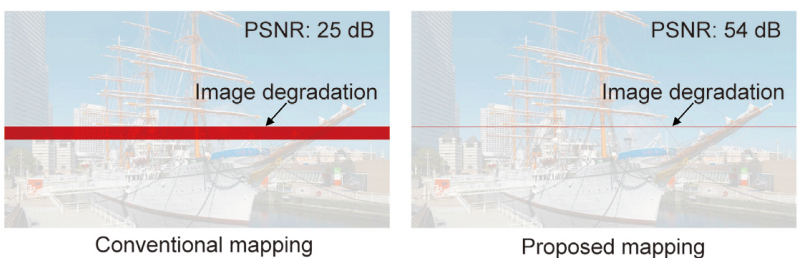

(c) Simulation results

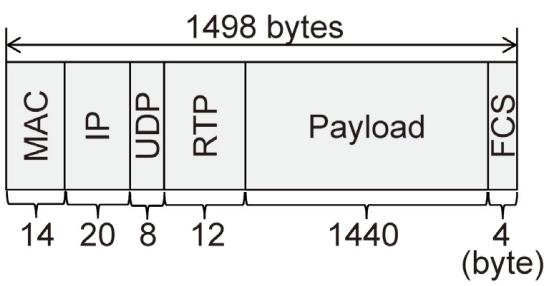

(d) Configuration as Ethernet frame

Fig.4 Packet mapping

whereas the conventional mapping generates 20736 packets per frame. The increase of packets is $0.18 \%$. Figure 4(c) shows simulation results of a packet lost on the slice boundary both the conventional mapping and the proposed mapping. In the conventional mapping, if the packet lost on the slice boundary, image degradation is observed wide-range in the picture. In this case, PSNR was equal to $25 \mathrm{~dB}$. On the other hand, in the proposed mapping, image degradation is observed narrow-range. In this case, PSNR was equal to $54 \mathrm{~dB}$. As these results, it has been confirmed that the proposed mapping method can reduce image degradation due to the packet lost on the slice boundary.

These mapping signals are configured as an Ethernet frame (Fig. 4(d)) after adding an RTP/UDP/IP header as an RTP payload. The RTP payload has a fixed length of 


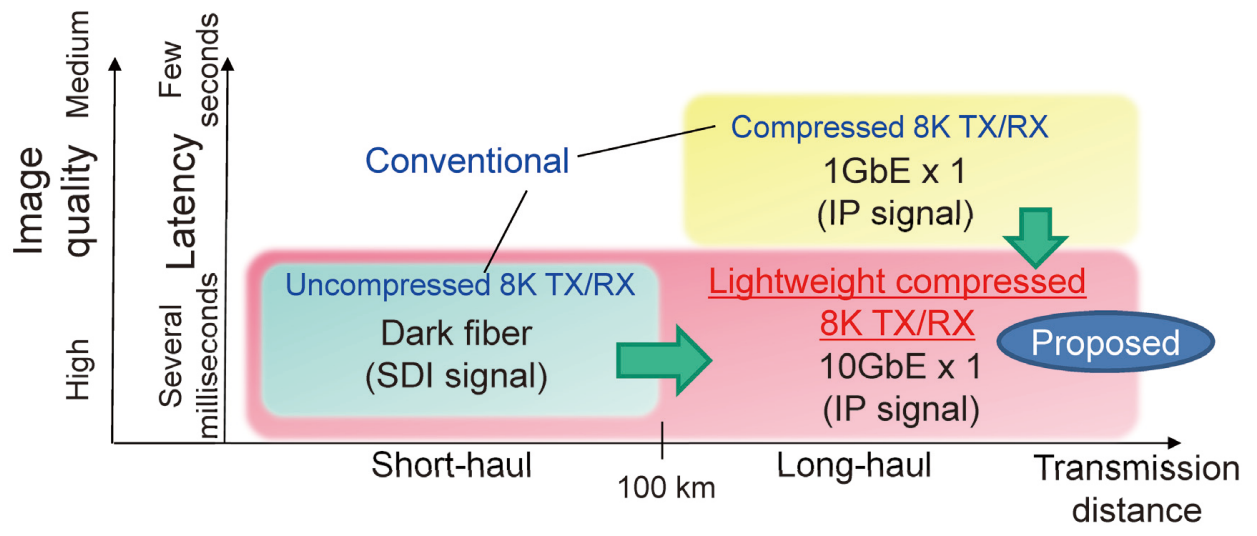

Fig.5 Application area of proposed device for $8 \mathrm{~K}$ TX/RX

1440 bytes.

\subsection{Synchronization between Transmitter and Receiver}

In SDI transmission, a slave synchronization system that can recover a clock from the received SDI signal on the receiver is used. In contrast, in IP transmission, since transceivers are generally asynchronous, transmitter devices require a mechanism to synchronize the input SDI signal clock on the transmitter side and the output SDI signal clock on the receiver side. In this work, precision time protocol (PTP) is adopted as the synchronization method. PTP is an inter-transmitterreceiver synchronization protocol over IP transmission, which was standardized as IEEE $1588^{30}$. It consists of a master/slave configuration, and it is possible to synchronize a time information of the PTP slave device by bidirectionally communicating the time information between the PTP master device and the PTP slave device. Both the transmitter and the receiver device function as PTP slave devices, and the transmitter and receiver are synchronized on the basis of the PTP signal input from the external PTP master device. In addition to PTP, black-burst and others can be input as synchronization signals.

\subsection{Application Area of Proposed Device}

An application area of a lightweight compressed 8K over IP transmission device is shown in Fig. 5. In the conventional $8 \mathrm{~K}$ live program production, two different transmission devices are used depending on the transmission distance from the venue to the broadcast station. Specifically, an uncompressed $8 \mathrm{~K}$ over SDI transmission device is used for transmission up to about $100 \mathrm{~km}$, and at distances beyond that, a compressed $8 \mathrm{~K}$ over IP transmission device is used. In the latter case, $8 \mathrm{~K}$ video is compressed to about several hundred $\mathrm{Mb} / \mathrm{s}$ by using a very large compression ratio such as high efficiency video coding (HEVC) ${ }^{31}$. A $1-\mathrm{GbE}$ line is used for the compressed $8 \mathrm{~K}$ over IP transmission device.
Although the uncompressed 8K over SDI transmission device can transmit $8 \mathrm{~K}$ program material with high image quality and low delay, using SDI signal transmission means that the line (e.g., communication line) has to be prepared separately from the video line. In the compressed $8 \mathrm{~K}$ over IP transmission device, degradation of image quality occurs due to compression and the processing latency time of several seconds, so it is difficult to share high-quality video in real time between the venue and the broadcast station.

In contrast, the proposed lightweight compressed $8 \mathrm{~K}$ over IP transmission device can transmit $8 \mathrm{~K}$ video with high image quality and low latency by using a relatively inexpensive $10-\mathrm{GbE}$ line regardless of the transmission distance. This device makes it possible to fulfill the two requirements for remote production, and in turn enables the production of $8 \mathrm{~K}$ live programs from broadcast stations, which had been nearly impossible until now.

\section{Experiments}

\subsection{Multi-channel Audio Remote Production with 8K Video}

In April 2018, remote production of multi-channel audio was carried out by using the lightweight compressed $8 \mathrm{~K}$ over IP transmission device. The remote production outline at that time is shown in Fig. 6(a). Two transmission lines were prepared in consideration of the redundant system of the $10-\mathrm{GbE}$ line and transmitted with the fiber length of about $20 \mathrm{~km}$. The $8 \mathrm{~K} 60 \mathrm{p}$ video signal delay between the lightweight compressed 8K over IP transmission device input and output was less than 1 millisecond, and the 192-ch audio signal delay was less than 3 milliseconds. Audio was delayed more than video due to the buffering of equipment that converts audio to IP. Both video and audio could be transmitted from the venue to the broadcast station with one video frame delay or less. As a result, we could successfully perform remote 


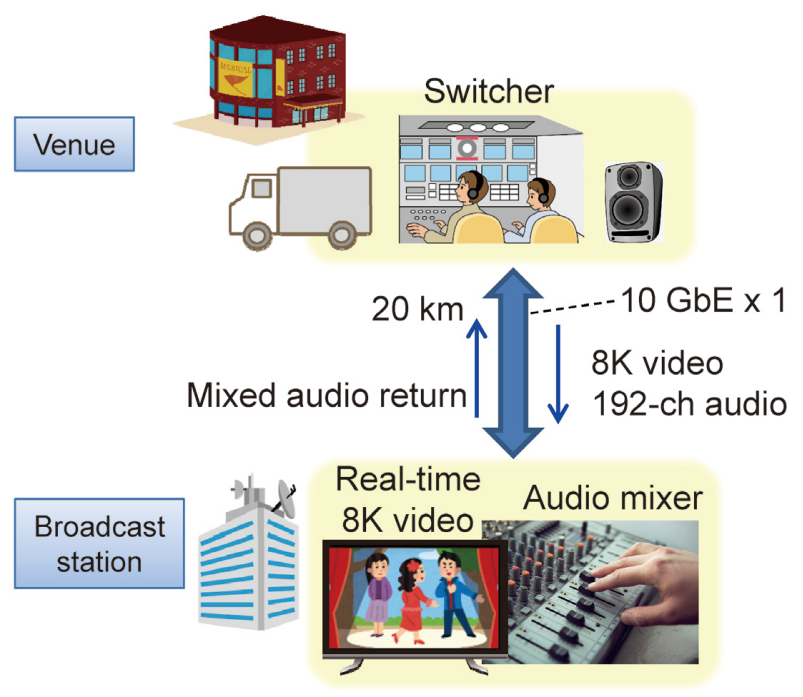

(a) Remote production outline

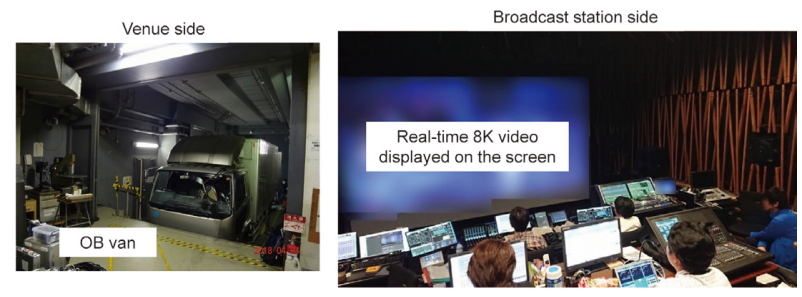

(b) Remote production experiment

Fig.6 Multi-channel audio remote production with $8 \mathrm{~K}$ video

production where audio mixing was carried out at the broadcast station while watching the venue's real-time 8K high-quality video.

Up until now, we had dispatched an audio mixing OB van for each venue, so we needed to set up the equipment for each site. Figure 6(b) shows the state of the remote production experiment. The results of this experiment showed that, even if a space was very narrow at the venue, it was possible to produce programs using the existing studio of the broadcast station. Therefore, it was possible to secure sufficient working space compared with program production at the venue. Production engineers involved in the experiment reported that they could set up the equipment in a shorter amount of time and that system debugging was easy. Overall, there were many favorable reactions to the new approach.

\subsection{Real-time 8K Camera Control from 1000-km IP Network}

We conducted a $1000-\mathrm{km}$ IP network transmission experiment using the lightweight compressed $8 \mathrm{~K}$ over IP transmission device in May 2018 at an NHK STRL open house. The system diagram is shown in Fig. 7(a). The video output from the $8 \mathrm{~K} 60 \mathrm{p}$ camera installed at the venue was compressed with the lightweight compressed $8 \mathrm{~K}$ over IP transmission device, which had a compression ratio of $1 / 5$, and transmitted for $1000 \mathrm{~km}$ with one 10-GbE line, and the de-compressed video was displayed on the $8 \mathrm{~K}$ monitor at the broadcast station. In the figure, blue lines indicate IP signals. All switches used are commercial off the shelf (COTS) switches, and boundary clock or transparent clock functions of PTP are not used. QoS control that gives priority to PTP signals was applied to edge switches. The transmission line was packet transferred by multi-protocol label switching (MPLS) using a commercial IP network provided by Internet Initiative Japan (IIJ) Inc. The transmission line consisted of a main line in the bandwidth of $100 \mathrm{GbE}$, and the $8 \mathrm{~K}$ traffic transmitted in this demonstration was multiplexed on the 100-GbE line together with traffic generated by a communication service (mobile for IIJ general purpose). The set-up of the demonstration is shown in Fig. 7(b). Figure 7(c) shows the timeline of each event. The network trip time was about $10 \mathrm{~ms}$ and the processing latency of the lightweight compressed $8 \mathrm{~K}$ over IP transmission device was about $1 \mathrm{~ms}$. The totaldelay in a single direction was about $11 \mathrm{~ms}$, which was sufficiently lower than one video frame. In addition, this time we remotely controlled one camera. In a use-case where multiple cameras at the venue are selected from the broadcast station, the selected camera signal is lightweight compressed and transmitted over IP. Therefore, the total-delay in a single direction does not change, and the video can be shared almost in real time.

Figure 8(a) shows the traffic fluctuation result (direction from the venue to the broadcast station). For the measurement, the Simple Network Management Protocol (SNMP) was used to record the response result of querying the data rate at intervals of 20 seconds for the switch at the broadcast station. The value obtained by averaging this inquiry result for 5 minutes is plotted. It is understood that a stream of about $8.3 \mathrm{~Gb} / \mathrm{s}$ was constantly transmitted at all times. Figure 8(b) shows an expanded graph of Fig. 8(a). The minimum data rate was $8.23 \mathrm{~Gb} / \mathrm{s}$, the maximum was $8.32 \mathrm{~Gb} / \mathrm{s}$, and the fluctuation was about $100 \mathrm{Mb} / \mathrm{s}$. The $8 \mathrm{~K}$ camera remote controller on the broadcast station side controlled the camera at the venue $1000 \mathrm{~km}$ away, and the $8 \mathrm{~K}$ video with the control was immediately reflected on the $8 \mathrm{~K}$ monitor in real time. The venue camera could be controlled from the broadcast station side without any feeling of discomfort.

PTP was used for synchronization between the broadcast station and the venue over network. Based on the PTP master installed at the broadcast station side, the PTP slave on the venue side synchronizes the time 


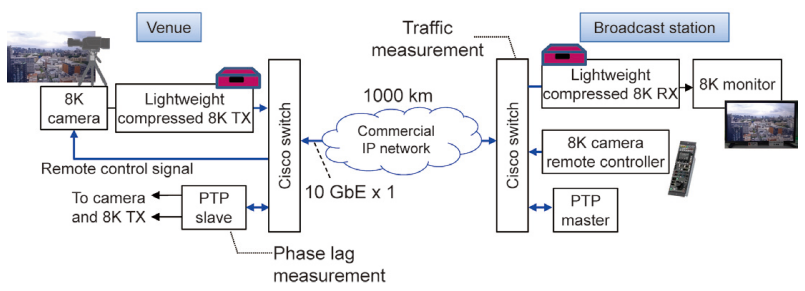

(a) System diagram

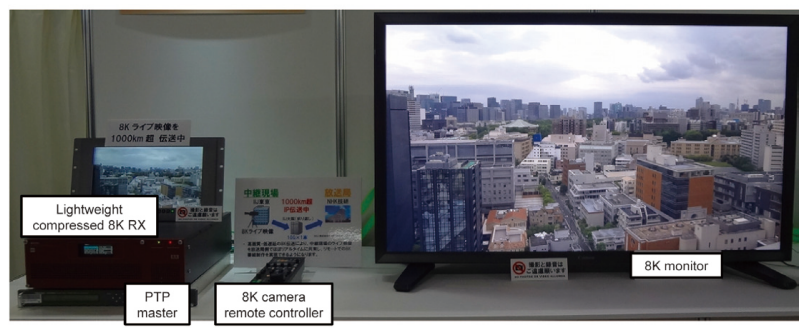

(b) Experiment (broadcast station side)

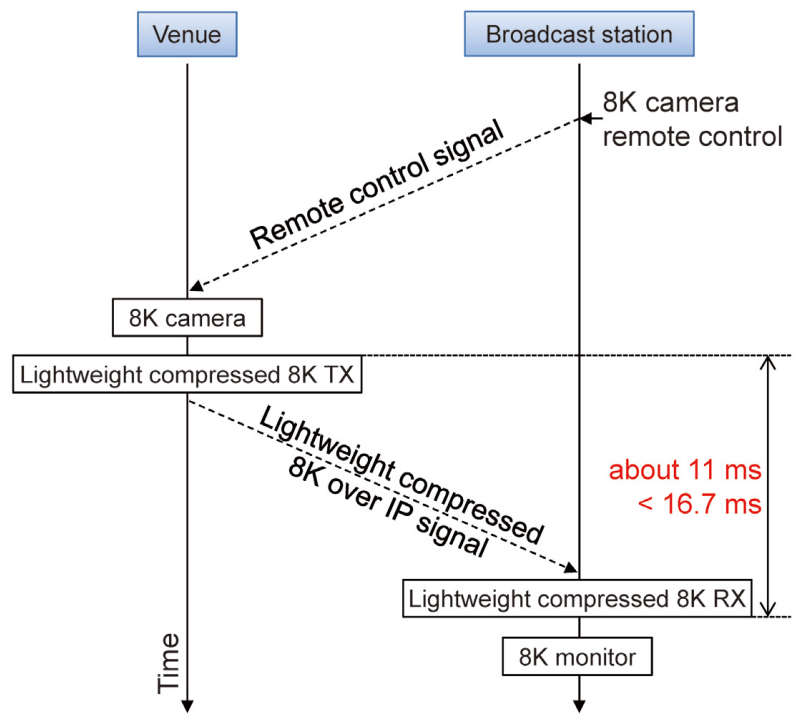

(c) Timeline

Fig.7 Remote production experiment over 1000-km IP network

and creates the frequency source for the video signal. Figure 8(c) shows a graph of the phase lag on the PTP slave side at the venue. Even in one-day operation, the lag was within -100 and $+300 \mathrm{~ns}$, which is sufficiently lower than the $1 \mu$ s indicated in a standard ${ }^{32}$, and stable video transmission could be achieved. The peak value of the phase lag was $281.79 \mathrm{~ns}$ (recorded at 4:37 a.m.) Similar trends are confirmed from measurement results every day. One of the fluctuations of the phase lag is thought to be due to asymmetry of traffic. Therefore, the reason the phase lag increases during the same period is that asymmetry of traffic occurs due to nightly maintenance (e.g., server update) in the main line section.

The lightweight $8 \mathrm{~K}$ over IP transmission device improved the processing delay from several seconds to 1 $\mathrm{ms}$ or less and the PSNR degradation to over $40 \mathrm{~dB}$ as compared with the existing compressed $8 \mathrm{~K}$ over IP

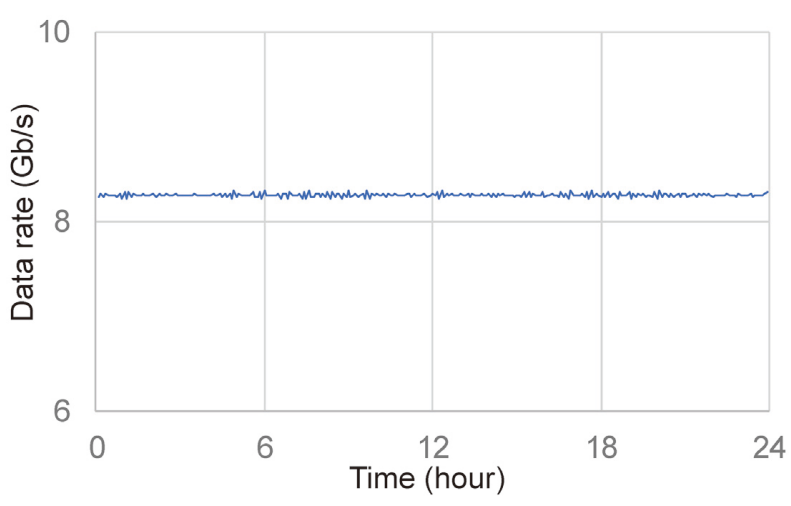

(a) Traffic from venue to broadcast station

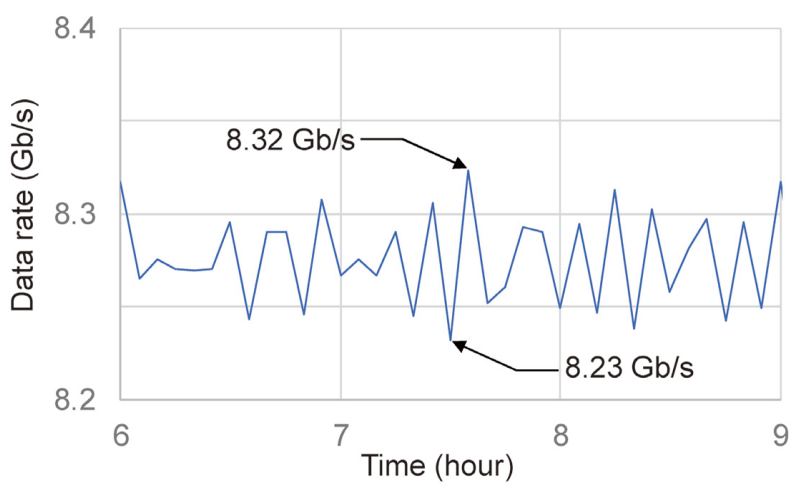

(b) Expansion of (a)

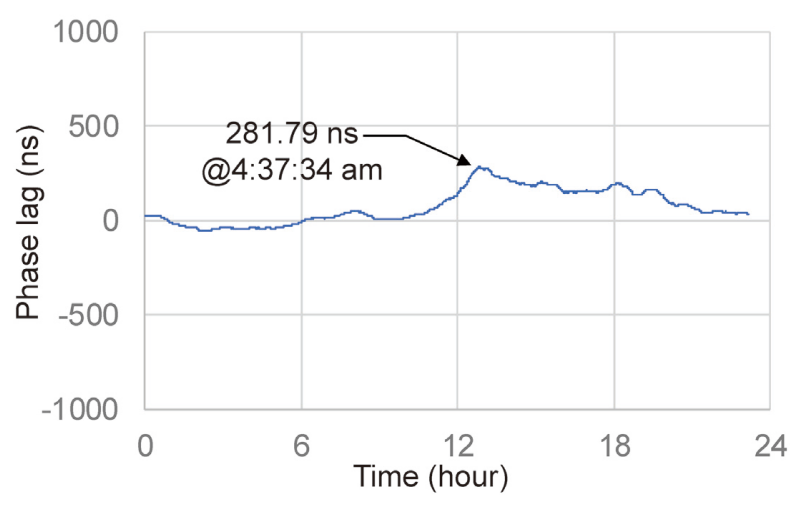

(c) Phase lag of PTP slave

Fig.8 Measurement results

transmission device. In addition, compared with the uncompressed $8 \mathrm{~K}$ over SDI transmission device, it also improved bandwidth with $10 \mathrm{GbE} \times 1$ and realized bidirectional communication using the IP signals. These results demonstrate that $8 \mathrm{~K}$ remote production can be realized even when the distance is $1000 \mathrm{~km}$ away by using the lightweight compressed $8 \mathrm{~K}$ over IP transmission device.

\section{Conclusion}

In this paper, we proposed a lightweight compressed $8 \mathrm{~K}$ over IP transmission device that achieves live remote production and discussed the results of experiments using this device. Results showed that the proposed 
device could approach realization of real-time $8 \mathrm{~K}$ remote production in live program production. In the future, we will study error correction codes suitable for lightweight compressed signals and endeavor to realize efficient and flexible program production using IP communication technology.

\section{References}

1) IEEE Std 802.3, "IEEE Standard for Ethernet".

2) D. Law, D. Dove, J. D'Ambrosia, M. Hajduczenia, M. Laubach, and S. Carlson, "Evolution of ethernet standards in the IEEE 802.3 working group," IEEE Communications Magazine, vol. 51, no. 8, pp. 88-96, 2013.

3) SMPTE ST 2022-6:2012, "Transport of High Bit Rate Media Signals over IP Networks (HBRMT)".

4) SMPTE ST 2110-10:2017, "Professional Media Over Managed IP Networks: System Timing and Definitions".

5) T. Edwards, "Studio Video over IP Progress Report," SMPTE Motion Imaging Journal, vol. 125, no. 7, pp. 85-87, 2016.

6) H. Yamaguchi and A. Lustica, "Audio and video over IP technology," 57th International Symposium ELMAR, pp. 125-128, 2015.

7) P. Elmer and H. Sariowan, "Interoperability for Professional Video Streaming over IP Networks," SMPTE Motion Imaging Journal, vol. 114, no. 2-3, pp. 81-85, 2005.

8) H. Stephansen and T. E. Krognes, "Experience with SDI Contribution over IP Network," SMPTE Motion Imaging Journal, vol. 116, no. 1, pp. 24-28, 2007.

9) ITU-R Recommendation BT.2020-1, "Parameter values for ultrahigh definition television systems for production and international programme exchange," 2014.

10) S. Hara, A. Hanada, I. Masuhara, T. Yamashita, and K. Mitani, "Celebrating the Launch of $8 \mathrm{~K} / 4 \mathrm{~K}$ UHDTV Satellite Broadcasting and Progress on Full-Featured 8K UHDTV in Japan," SMPTE Motion Imaging Journal, vol. 127, no. 2, pp. 1-8, 2018.

11) H. Gaggioni, M. Ueda, F. Saga, K. Tomita, and N. Kobayashi, "Serial Digital Interface for HDTV," SMPTE Motion Imaging Journal, vol. 106, no. 5, pp. 298-304, 1997.

12) SMPTE ST 292-2008, "1.5 Gb/s Signal/Data Serial Interface".

13) SMPTE ST 2082-1:2015, "12 Gb/s Signal/Data Serial Interface Electrical".

14) IEEE Std 802.3bs-2017, "Media Access Control Parameters, Physical Layers and Management Parameters for $200 \mathrm{~Gb} / \mathrm{s}$ and $400 \mathrm{~Gb} / \mathrm{s}$ Operation"

15) B. Johnston, "Taking Remote Production to the Next Level: CBC's Coverage of the 2014 Sochi Olympic Games," SMPTE Motion Imaging Journal, vol. 124, No.2, pp.1-12, 2015

16) E. Calverley, "Time-Compensated Remote Production Over IP," SMPTE Motion Imaging Journal, vol. 127, No.5, pp.51-57, 2018

17) M. Keltsch, S. Prokesch, O.P. Gordo, J. Serrano, T.K. Phan and I. Fritzsch, "Remote production and mobile contribution over 5G networks: Scenarios, requirements and approaches for broadcast quality media strieaming," 2018 IEEE International Symposium on Broadband Multimedia Systems and Broadcasting (BMSB), pp.1-7, 2018

18) H. Stephansen and T.E. Krognes, "Experience with SDI contribution over IP network," SMPTE Motion Imaging Journal, vol. 116, No.1, pp.24-28, 2007

19) M. Levy, L.R. Richardson and G. Rouvroy, "4K video over SMPTE 2022-5/6 workflows," SMPTE Motion Imaging Journal, vol. 125, No.2, pp.1-7, 2016

20) SMPTE ST 2082-12:2016, "4320-line and 2160-line Source Image and Ancillary Data Mapping for Quad-link 12G-SDI"

21) ITU-R Recommendation BT.2077-1, "Real-time serial digital interfaces for UHDTV signals," 2015

22) SMPTE RDD 34:2015, "LLVC - Low Latency Video Codec for Network Transfer"

23) SMPTE ST 2042-1:2017, "VC-2 Video Compression"
24) SMPTE RDD 35:2016, "TICO Lightweight Codec Used in IP Networked or in SDI Infrastructures"

25) ISO/IEC DIS 21122-1: 2019, "Information technology - JPEG XS low-latency lightweight image coding system - Part 1: Core coding system"

26) IETF WG Document, "RTP Payload Format for ISO/IEC 21122 (JEPG XS)," Web site: https://datatracker.ietf.org/doc/draft-ietfpayload-rtp-jpegxs/

27) The Institute of Image Information and Television Engineers, "Ultra-high definition/wide-color-gamut standard test sequences Series B". Available: https://www.ite.or.jp/contents/chart/uhdtv_b/ UHDTV_Bseries_manual.pdf

28) J. Kawamoto, T. Koyama, M. Kawaragi, T. Kurakake and K. Saito, "Remote Production Experiments with Lightweight Compressed 8K UHDTV over IP Device," 2018 Principles, Systems and Applications of IP Telecommunications (IPTComm), no.3, pp.1-7, 2018

29) J. Kawamoto and T. Kurakake, "XOR-Based FEC to Improve Burst-Loss Tolerance for 8K Ultra-High Definition TV over IP Transmission," 2017 IEEE Global Communications Conference (GLOBECOM), CSSSMA.4-05, 2017

30) IEEE Std 1588-2008, "IEEE Standard for a Precision Clock Synchronization Protocol for Networked Measurement and Control Systems"

31) G.J. Sullivan, J.-R. Ohm, W.-J. Han and T. Wiegand, "Overview of the high efficiency video coding (HEVC) standard," IEEE Trans. Circuits Syst. Video Technol., 22, 12, pp.1649-1668, 2012

32) SMPTE ST 2059-2:2015, "SMPTE Profile for Use of IEEE-1588 Precision Time Protocol in Professional Broadcast Applications"

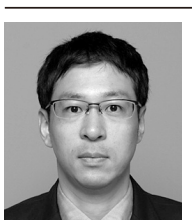

Junichiro Kawamoto received M.E. and $\mathrm{Ph} . \mathrm{D}$. degrees from the University of Tsukuba, Ibaraki, Japan in 2002 and 2017, respectively. He joined Japan Broadcasting Corporation (NHK) in 2008. He has mainly been engaged in the research and development of $4 \mathrm{~K} / 8 \mathrm{~K}$ UHDTV program production system using IPnetworks.

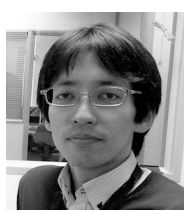

Tomofumi Koyama received a M.E. degree in Tokyo Institute of Technology, Tokyo, Japan. He joined Japan Broadcasting Corporation (NHK) in 2005. Since 2009, He has been engaged in the research and development of transporting media for program production over IP-networks.

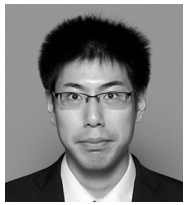

Masahiro Kawaragi received his B.E. and M.E. degrees in computer science from the Toyohashi University of Technology, Toyohashi, Japan, in 2012 2014. He joined Japan Broadcasting Corporation (NHK) in 2014. Since 2017, he has been engaged in research on IP based live production systems at NHK Science and Technology Research Laboratories.

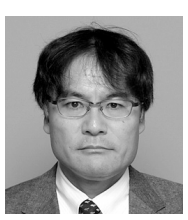

Kyoichi Saito received the B.E. degree in Electrical Engineering from Keio University, Yokohama, Japan in 1989. He has been with Japan Broadcasting Corporation(NHK) since 1989. He has been engaged in research on multiplexing scheme of digital broadcasting, and in system design and development of digital broadcasting systems.

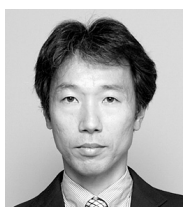

Takuya Kurakake received B.E. and M.E. degrees from the University of Tokyo, Tokyo, Japan, and a Ph.D. degree from the University of ElectroCommunications, Tokyo, Japan, in 1992, 1994, and 2014, respectively. He joined Japan Broadcasting Corporation (NHK) in 1994. He has mainly been engaged in the research and development of optical transmission systems and digital transmission systems for cable television, and 4K/8K UHDTV program production system using IP-networks. 